\title{
Serial proton magnetic resonance spectroscopy in a patient with the interval form of carbon monoxide poisoning
}

Tetsuhito Murata, Satoshi Itoh, Yoshifumi Koshino, Masao Omori, Ichiro Murata, Kazumasa Sakamoto, Kiminori Isaki, Hirohiko Kimura, Yasushi Ishii

\begin{abstract}
Serial proton magnetic resonance spectroscopy ('H-MRS) studies were performed from immediately after the appearance of sequelae in a patient with the interval form of carbon monoxide (CO) poisoning. The volume of interest was set over the frontal lobe white matter. In the early period a persistent increase in choline was found, which was thought to reflect the course of progressive demyelination. The appearance of lactate and decrease in $\mathbf{N}$-acetylaspartate reflected the point at which neuron injury became irreversible. These were followed later by the finding of irreversible changes on MRI and single photon emission computed tomography. The findings suggest that ${ }^{1} \mathrm{H}-\mathrm{MRS}$ may be a useful modality to determine neuron viability and prognosis early in the course of the interval form of $\mathrm{CO}$ poisoning.
\end{abstract}

$(\mathcal{F}$ Neurol Neurosurg Psychiatry 1995;58:100-103)

Keywords: carbon monoxide poisoning; proton magnetic resonance spectroscopy; magnetic resonance imaging

The interval form of carbon monoxide (CO) poisoning is characterised by disturbed consciousness in the acute phase, then a period of several days to several weeks in which no clinical symptoms are apparent, after which neurological or psychiatric symptoms reappear. ${ }^{1}$ Progressive demyelination in the white matter seems to be the main pathological feature associated with this course, ${ }^{2}$ and has been noted in $0.06 \sim 2.8 \%$ of all patients with $\mathrm{CO}$ poisoning. ${ }^{3}$

We describe a patient with the interval form of $\mathrm{CO}$ poisoning in whom serial proton magnetic resonance spectroscopy ( $\left.{ }^{1} \mathrm{H}-\mathrm{MRS}\right)$ studies were conducted from immediately after the onset of sequelae to investigate changes in brain metabolic activity. The volume of interest was set over the frontal lobe white matter. Single photon emission computed tomography (SPECT) and MRI were performed at the same site to clarify the relation between these changes in brain metabolic activity and changes in brain morphology and blood flow.

\section{Case report}

The patient was a 57 year old man whose family and medical histories were non-contributory. Around noon on 9 February 1993 the patient and his wife were exposed to $\mathrm{CO}$ gas while heating red beans on a briquet brazier in a closed room, and lost consciousness. About six hours later the patient regained consciousness, but his wife remained unconscious and did not answer when her name was called. The patient called for an ambulance, which took his wife to the emergency department of a nearby hospital. Carbon monoxide poisoning was not initially suspected and because of a history of myocardial infarction, as well as the results of blood biochemical and electrocardiographic examinations, the wife was suspected of having had a second infarction. Subsequently, the patient continued his normal occupation and also helped to look after his wife in hospital. From around 20 March (the 39th day after exposure) the patient suddenly became sluggish in his daily activities, and often showed mutism and a vacant air even while caring for his wife. He was disoriented with regard to date and place. On 29 March (the 48th day after exposure) the patient presented to our department. On neurological examination, primitive reflexes (grasp, palmomental reflexes) were noted, but no other pathological reflexes or abnormal deep reflexes were found. Because of difficulty in standing up, walking was impossible, and he was incontinent of urine and faeces. No particular abnormalities were noted on blood biochemical examinations. From the history the interval form of $\mathrm{CO}$ poisoning was suspected.

From the second admission day (the 49th day after exposure) hyperbaric oxygen treatment and cerebral metabolism enhancing agents were given. From about one week after admission (the 55th day after exposure) speech was almost absent. On 12 April (the 62nd day after exposure) a feeding gastrostomy was established, because he did not swallow even when liquid and solid foods were placed in his mouth. From the end of April (about the 80th day after exposure) no voluntary movement, except for that of the eyes, was noted. At present, 180 days after exposure, the patient continues to show an apallic syndrome. 
Figure 1 Serial changes in the present patient in the relative area ratio of each peak to the total area of the four peaks ( $\mathrm{Lac}+$ $N A A+C r+C h o)$ namely, Choltotal, NAA/total, and Lac/total. See text for details. Mean (2SD) of Choltotal and $N A A /$ total in nine normal subjects aged in their $50 \mathrm{~s}$ are given. No Lac peak was found in any of the normal subjects and thus Lac/total was zero.

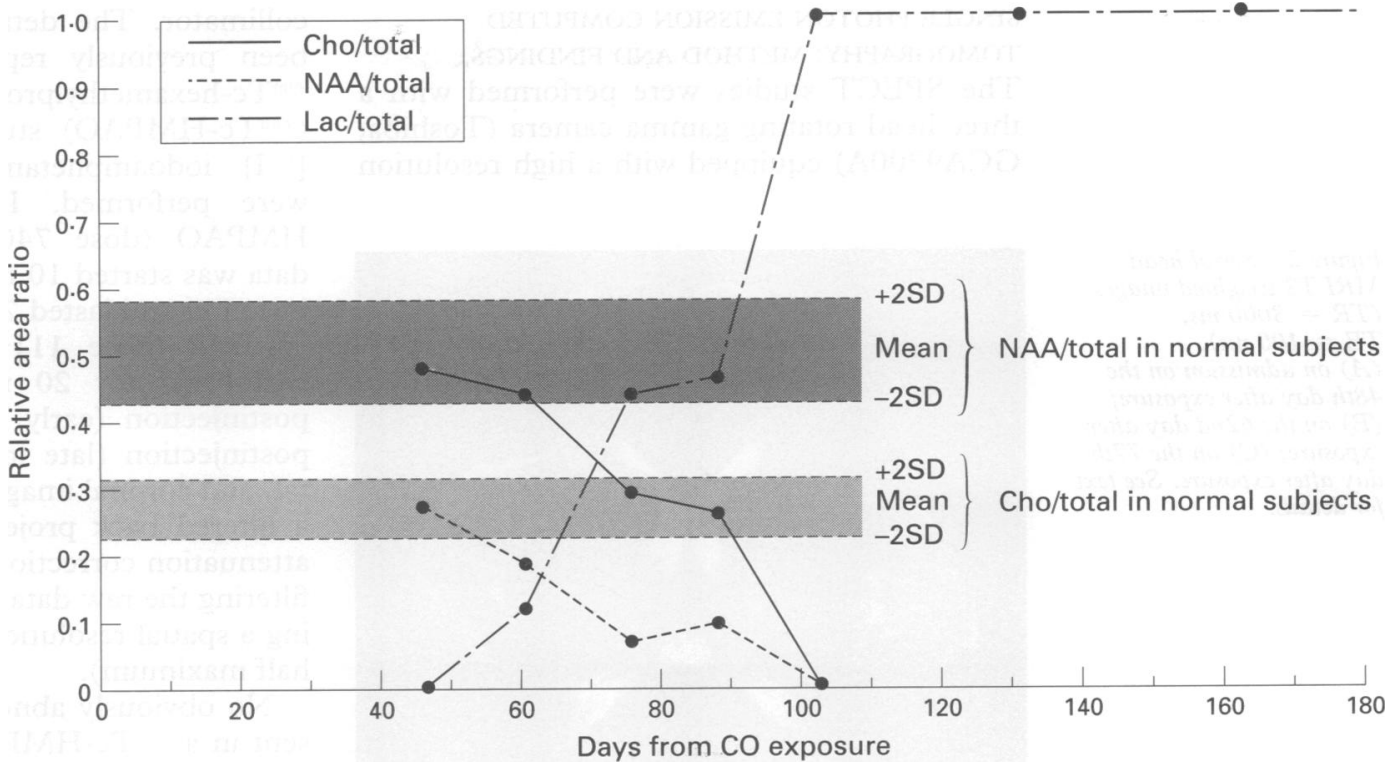

SERIAL PROTON MAGNETIC RESONANCE SPECTROSCOPY: METHOD AND FINDINGS The method was that used in our previous report, ${ }^{4}$ and it is described only briefly here. The MRI investigations were performed on a conventional 1.5 Tesla imager (SIGNA,GE) with a standard head coil (linear birdcage resonator). Volume selection was achieved by stimulated echo acquisition mode (STEAM) sequence. The ${ }^{1} \mathrm{H}-\mathrm{MR}$ spectra were acquired with a repetition time (TR) of $2000 \mathrm{~ms}$, echo time (TE) of $272 \mathrm{~ms}$, and mixing time (TM) of $21.6 \mathrm{~ms}$. Water suppression was achieved by chemical shift-selective (CHESS) pulses followed by dephasing gradients in the preparation periods. A volume of interest measuring $2 \times 2 \times 2 \mathrm{~cm}^{3}$ was selected in the left frontal lobe white matter around the anterior horn of the lateral ventricle. Then, one neuroradiologist (SI) blind to the other data calculated the peak area ratios of $\mathrm{N}$-acetylaspartate (NAA), total creatine (Cr), choline containing compounds (Cho), and lactate (Lac) from the spectra with a curve fitting method using the mixture ratio of Gaussian and Lorenzian curves (Gaussian: 50-70\%, Lorenzian: $30-50 \%$ ). Area measurement was performed manually with the GENCAP software program on a 1280 Data Station.

Figure 1 shows the serial changes in this patient in the relative area ratio of each peak to the total area of the four peaks (Lac + NAA + Cr + Cho)-namely, Cho/ total, NAA/total, and Lac/total. In a group of nine normal subjects aged in their 50 s (mean 56.3 (SD 2.6) years), the means (SD) of Cho/total and NAA/total were $0.28(0.02)$ and $0.51(0.04)$ respectively, with the respective mean values and range of mean values (2SD) indicated in fig 1 . No lactate peak was found in any of the normal subjects and thus Lac/total was zero. In the present patient, Cho/total was 0.49 and 0.46 on the 48 th and 62 nd days after exposure respectively, clearly exceeding in both cases the mean (2SD) of the normal subjects. On the 77th and 90th days after exposure these values decreased to close to the mean values of the normal subjects, and from the 104th day after exposure became zero as the Cho peak disappeared. The NAA/total was 0.28 on the 48 th day after exposure, representing a value much lower than the mean (2SD) of the normal group. Subsequently, this value further decreased and from the 104th day after exposure became zero as the NAA peak disappeared. Lac/total was initially zero, and gradually increased from the 62 nd day after exposure when a Lac peak first appeared. As only the Lac peak persisted from the 104th day after exposure when the Cho and NAA peaks both disappeared, the value of Lac/total was consistently 1.0 after this time.

MAGNETIC RESONANCE IMAGING FINDINGS

T2 weighted images obtained on the 48th day after exposure showed symmetric slightly hyperintense areas in the frontal and temporoparietal deep white matter (fig 2 (A)), whereas no abnormal findings were evident on $\mathrm{T} 1$ weighted images of the same sites. The symmetric hyperintense areas were somewhat enlarged on T2 weighted images obtained on the 62nd day after exposure, in particular in the bilateral frontal lobe deep white matter (fig 2 (B)). Although no pronounced changes were evident on T1 weighted images, symmetric slightly hypointense areas in the frontal lobe white matter were seen in the centre of the hyperintense areas noted on the T2 weighted images. On T2 weighted images obtained on the 77th day after exposure the extent and signal intensity of the lesions in the white matter were increased, but no enlargement of the ventricles was seen (fig 2 (C)). On T1 weighted images the symmetric hypointense areas in the frontal lobe white matter were enlarged. On $T 1$ and $T 2$ weighted images obtained on the 164th day after exposure, the lateral and third ventricles were very enlarged, whereas there was no appreciable change in the extent of the white matter lesions. 
SINGLE PHOTON EMISSION COMPUTED

TOMOGRAPHY: METHOD AND FINDINGS

The SPECT studies were performed with a three head rotating gamma camera (Toshiba, GCA9300A) equipped with a high resolution

Figure 2 Serial head $M R I T 2$ weighted images $(T R=3000 \mathrm{~ms}$, $T E=100 \mathrm{~ms}$ )

(A) on admission on the 48th day after exposure;

(B) on the 62nd day after exposure; (C) on the 77th day after exposure. See text for details.
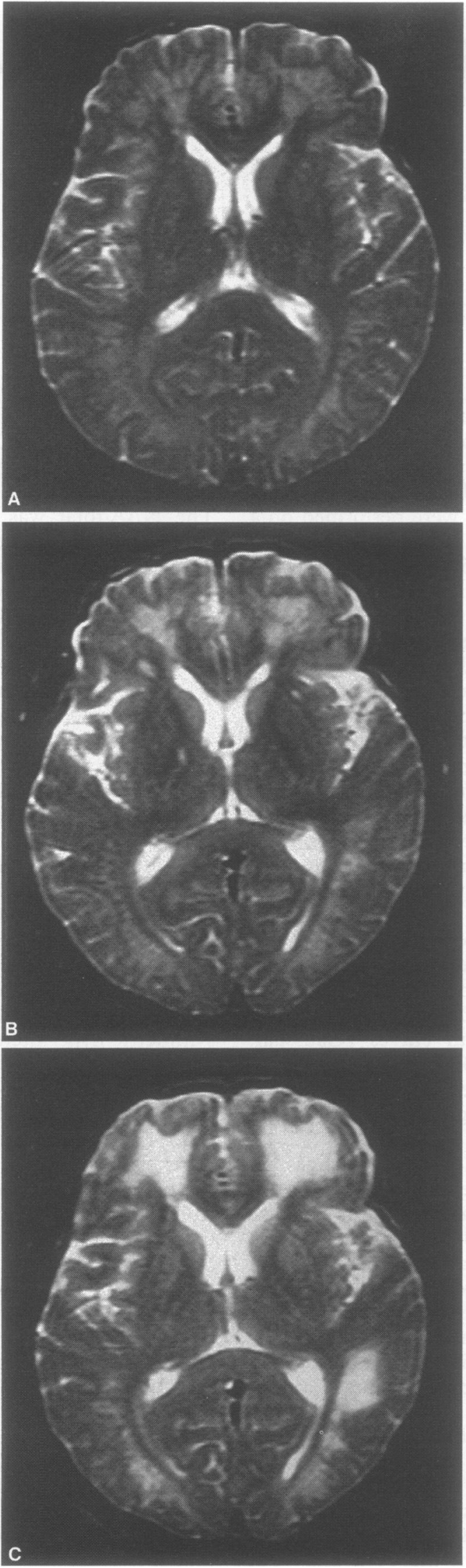

collimator. The details of the method have been previously reported. ${ }^{5}$ After an initial ${ }^{99 \mathrm{~m}} \mathrm{Tc}$-hexamethylpropyleneamine oxime ( ${ }^{99}$ Tc-HMPAO) study, two $\mathrm{N}$-isopropyl-p[ $\left.{ }^{123} \mathrm{I}\right]$ iodoamphetamine ( $\left.{ }^{123} \mathrm{I}-\mathrm{IMP}\right)$ studies were performed. In the case of ${ }^{99 \mathrm{~m} T c}$ HMPAO (dose $740 \mathrm{MBq}$ ), acquisition of data was started 10 minutes after intravenous injection and lasted 20 minutes. In the case of ${ }^{123}$ I-IMP (dose $111 \mathrm{MBq}$ ) acquisition was performed for 20 minutes at 20 minutes postinjection (early image) and three hours postinjection (late image). Transaxial, sagittal, and coronal images were reconstructed by a filtered back projection algorithm without attenuation correction (Ramp filter) after prefiltering the raw data (Butterworth filter), giving a spatial resolution of $8 \mathrm{~mm}$ (full width at half maximum).

No obviously abnormal findings were present in a ${ }^{99 m}$ Tc-HMPAO study performed on the 49th day after exposure (day after admission), although the possibility of slightly decreased perfusion in the frontal white matter could not be excluded. On a ${ }^{123}$ I-IMP study performed on the 64th day after exposure early images showed a widespread reduction in perfusion in the cerebral cortex and white matter, in particular in the frontal and parieto-occipital lobes, whereas delayed images did not show reduced perfusion in these areas. No abnormal findings were noted in the thalamus or basal ganglia. Early images on a ${ }^{123}$ I-IMP study performed on the 111th day after exposure showed greatly reduced perfusion in the cerebral cortex and white matter, in particular in the frontal and parieto-occipital lobes. Delayed images also showed reduced perfusion in the bilateral frontal lobes, especially in the white matter.

\section{Discussion}

In the present ${ }^{1} \mathrm{H}$-MRS studies with the volume of interest set over the frontal lobe white matter the following abnormal spectral patterns were found: a relative increase in choline containing compounds, a relative decrease in $\mathrm{N}$-acetylaspartate, and the appearance of lactate. The peak of choline containing compounds reflects the amount of choline containing substances such as phosphocholine and glycerophosphocholine, which play a part in membrane metabolism ${ }^{6}$ and have been reported to fluctuate when membranes are degraded or rapidly synthesised. ${ }^{7}$ Choline containing compounds in the present patient were persistently raised from immediately after the appearance of sequelae, during which period the MRI findings suggested progressive demyelination of the white matter. This implies that in the interval form of $\mathrm{CO}$ poisoning the increase in choline containing compounds reflects a process of active, progressive demyelination occurring in the white matter.

$\mathrm{N}$-Acetylaspartate is thought to be present only in nerve cells, ${ }^{8}$ and when decreased reflects loss or degeneration of neurons. ${ }^{9}$ In 
the present patient, a decrease in $\mathrm{N}$-acetylaspartate was found from the initial examination, suggesting that loss or degeneration of neurons were already occurring immediately after the appearance of sequelae. Subsequently, the relative decrease in $\mathrm{N}$ acetylaspartate persisted even while the increase in choline containing compounds continued, suggesting the possibility that secondary neuron injury may have been augmented by progressive demyelination.

Lactate is produced by anaerobic glycolysis when the oxidation derived supply of adenosine triphosphate is inadequate under conditions of ischaemia or hypoxia. In the present patient, lactate first appeared more than three weeks after the appearance of sequelae, when demyelination and the accompanying nerve cell injury were fairly advanced, and gradually increased thereafter.

The prognosis of the interval form of $\mathrm{CO}$ poisoning has been variously reported to be relatively benign, or unfavourable with a relentlessly progressive course, ${ }^{10}$ and few markers are available to predict clearly the course in individual patients. In the present case, clear abnormalities in brain metabolic activity (increase in choline containing compounds and decrease in $\mathrm{N}$-acetylaspartate) were already apparent on ${ }^{1} \mathrm{H}-\mathrm{MRS}$ immediately after the appearance of sequelae, in the period in which few prognostically relevant findings were noted on MRI or SPECT. The appearance of lactate and the period of its persistence as well as the degree of the decrease in $\mathrm{N}$-acetylaspartate may also be useful markers reflecting neuron viability and prognosis in the interval form of $\mathrm{CO}$ poisoning.

1 Ginsberg MD. Delayed neurological deterioration following hypoxia. In: Fahn S, Davis JN, Rowland LP, eds. Advances in neurology. Vol 26. Cerebral hypoxia and it consequences. New York: Raven Press, 1979:21-44.

2 Chang KH, Han MH, Kim HS, Wie BA, Han MC. Delayed encephalopathy after acute carbon monoxide intoxication: MR imaging features and distribution of cerebral white matter lesions. Radiology 1992;184 117-22.

3 Choi IS. Delayed neurologic sequelae in carbon monoxide intoxication. Arch Neurol 1983;40:433-5.

4 Murata T, Koshino Y, Omori M, et al. In vivo proton magnetic resonance spectroscopy study on premature aging in adult Down's syndrome. Biol Psychiatry 1993;

5 Matsuda H, Oskoie SD, Kinuya $\mathrm{K}$, et al. Tc-99m HMPAO brain perfusion tomography atlas using a high resolution

6 Van der Knaap MS, van der Grond J, van Rijen PC, Faber JAJ, Valk J, Willemse K. Age dependent changes in localized proton and phosphorus MR spectroscopy of the brain. Radiology 1990;176:509-15.

7 Dawson RMC. Enzymatic pathways of phospholipid metabolism in the nervous system. In: Eichberg J, ed. Phospholipids in nervous tissues. New York: John Wiley and Sons Inc, 1985:45-78.

8 Nadler JV, Cooper JR. N-acetyl-L-aspartic acid content of human neural tumours and bovine peripheral nervous tissues. $\mathcal{F}$ Neurochem 1972;19:313-9.

9 Menon DK, Sargentoni J, Peden CJ, et al. Proton MR spectroscopy in herpes simplex encephalitis: assessment of neuronal loss. $\mathcal{F}$ Comput Assist Tomogr 1990;14: 449-52.

10 Vieregge $P$, Klostermann $W$, Blümm RG, Borgis $\mathrm{KJ}$. Carbon monoxide poisoning: clinical, neurophysiologi$\mathrm{cal}$, and brain imaging observations in acute disease and follow-up. F Neurol 1989;236:478-81. 